\title{
Space Mapping with Distributed Fine Model Evaluation for Optimization of Microwave Structures and Devices
}

\author{
Slawomir Koziel, Senior Member, IEEE, and John W.Bandler, Life Fellow, IEEE \\ School of Science and Engineering, Reykjavik University, Kringlunni 1, IS-103 Reykjavik, Iceland \\ Department of Electrical and Computer Engineering, McMaster University, Hamilton, ON, Canada L8S 4K1
}

\begin{abstract}
A new space mapping optimization algorithm for microwave design is presented. We implement a distributed fine model evaluation through independent processing of the fine model responses corresponding to consecutive frequency samples using a number of processors. This allows us to obtain a substantial reduction of the overall optimization time for the space mapping algorithm. When our technique is used together with previously published methods of reducing the computational cost of solving the parameter extraction and surrogate optimization sub-problems, the total optimization time of the microwave structure can be comparable to or less than a single fine model evaluation on a single processor. Illustration examples are provided.
\end{abstract}

Index Terms - Computer-aided design (CAD), EM optimization, space mapping, surrogate modeling, parallel model evaluation.

\section{INTRODUCTION}

Space mapping (SM) addresses the problem of optimization of expensive functions, also called "fine" models, through iterative optimization and updating of the surrogate models which are built using cheaper "coarse" models [1]-[3]. In the microwave area, the "fine" model is typically implemented with a high fidelity CPU-intensive EM simulator. The "coarse" model can be an equivalent circuit of the corresponding device. SM proved to be successful in many engineering areas (e.g., [4]-[6]).

A lot of effort has been devoted to improving the efficiency of SM optimization. Recent work includes: (i) introducing new algorithms and SM surrogate model types in order to reduce the number of fine model evaluations necessary to find a satisfactory solution (e.g., [1]-[3]); (ii) improving convergence properties of SM algorithms (e.g., [7]); and (iii) reducing the computational overhead of the parameter extraction and surrogate optimization sub-problems [8], [9].

Here we present a new implementation of SM optimization with distributed evaluation of the fine model, realized through independent processing of the fine model responses

This work was supported in part by the Natural Sciences and Engineering Research Council of Canada under Grants RGPIN7239-06, STPGP33676006, and by Bandler Corporation.

S. Koziel was with the Simulation Optimization Systems Research Laboratory, Department of Electrical and Computer Engineering, McMaster University, Hamilton, ON, Canada L8S 4K1. He is now with the School of Science and Engineering, Reykjavik University, Kringlunni 1, IS-103 Reykjavik, Iceland.

J.W. Bandler is with the Simulation Optimization Systems Research Laboratory, Department of Electrical and Computer Engineering, McMaster University, Hamilton, ON, Canada L8S 4K1 and also with Bandler Corporation, Dundas, ON, Canada L9H 5E7. corresponding to consecutive frequency samples using a number of machines. This allows parallelization of the fine model processing regardless of whether the fine model simulator has a multi-processor analysis capability or not. Also, it allows us to use any mixture of PCs, workstations and nodes of the computational cluster, if available. The new algorithm has been implemented within the SMF system [10], [11].

\section{SPACE MAPPING OPTIMIZATION ALGORITHM}

Let $\boldsymbol{R}_{f}$ denote the response vector of a fine model of the device of interest, which might be the evaluation of some characteristics of the device, e.g., $\left|S_{21}\right|$, at a given set of frequencies. Our goal is to solve

$$
\boldsymbol{x}_{f}^{*}=\arg \min _{\boldsymbol{x}} U\left(\boldsymbol{R}_{f}(\boldsymbol{x})\right)
$$

where $U$ is a given objective function. We consider an optimization algorithm that generates a sequence of points $\boldsymbol{x}^{(i)}$, $i=0,1,2, \ldots$, and a family of surrogate models $\boldsymbol{R}_{s}^{(i)}$, so that

$$
\boldsymbol{x}^{(i+1)}=\arg \min _{\boldsymbol{x}} U\left(\boldsymbol{R}_{\mathrm{s}}^{(i)}(\boldsymbol{x})\right)
$$

Let $\boldsymbol{R}_{c}$ denote the response vector of the coarse model that describes the same object as the fine model: less accurate but much faster to evaluate. Surrogate models are constructed from the coarse model so that the misalignment between $\boldsymbol{R}_{s}^{(i)}$ and the fine model is minimized. $\boldsymbol{R}_{s}^{(i)}$ is defined as

$$
\boldsymbol{R}_{s}^{(i)}(\boldsymbol{x})=\overline{\boldsymbol{R}}_{s}\left(\boldsymbol{x}, \boldsymbol{p}^{(i)}\right)
$$

where $\overline{\boldsymbol{R}}_{s}$ is a generic SM surrogate model, i.e., the coarse model composed with suitable SM transformations.

$$
\boldsymbol{p}^{(i)}=\arg \min _{\boldsymbol{p}} \sum_{k=0}^{i} w_{i . k}\left\|\boldsymbol{R}_{f}\left(\boldsymbol{x}^{(k)}\right)-\overline{\boldsymbol{R}}_{s}\left(\boldsymbol{x}^{(k)}, \boldsymbol{p}\right)\right\|
$$

is a vector of model parameters and $w_{i . k}$ are weighting factors. A variety of SM surrogate models is available [1]-[3], e.g., the input SM [1], in which the generic SM surrogate model takes the form $\overline{\boldsymbol{R}}_{s}(\boldsymbol{x}, \boldsymbol{p})=\overline{\boldsymbol{R}}_{s}(\boldsymbol{x}, \boldsymbol{B}, \boldsymbol{c})=\boldsymbol{R}_{c}(\boldsymbol{B} \cdot \boldsymbol{x}+\boldsymbol{c})$. Typically, the starting point $\boldsymbol{x}^{(0)}$ of the SM optimization algorithm is a coarse model optimal solution, i.e., $\boldsymbol{x}^{(0)}=\arg \min \left\{\boldsymbol{x}: U\left(\boldsymbol{R}_{c}(\boldsymbol{x})\right)\right\}$.

The space mapping optimization algorithm flow can be described as follows:

Step 1 Set $i=0$;

Step 2 Evaluate the fine model to find $\boldsymbol{R}_{f}\left(\boldsymbol{x}^{(i)}\right)$;

Step 3 Obtain the surrogate model $\boldsymbol{R}_{s}^{(i)}$ using (3) and (4);

Step 4 Given $\boldsymbol{x}^{(i)}$ and $\boldsymbol{R}_{s}^{(i)}$, obtain $\boldsymbol{x}^{(i+1)}$ using (2);

Step 5 If the termination condition is not satisfied go to Step 2; else terminate the algorithm; 
Usually, the algorithm is terminated when it converges or when the maximum number of iterations is exceeded.

A reduction of the computational cost of SM optimization can be obtain through a reduction in the number of fine model evaluations, a reduction in the computational overhead of parameter extraction and surrogate model optimization, or by decreasing the evaluation time for the fine model. The first two options have undergone significant research recently as described in the introduction. The last possibility, described in the next section, can be realized by a distributed evaluation of the fine model.

\section{Distributed Evaluation Of THE Fine MODEL IN SMF}

A distributed evaluation of the fine model has been implemented within the SMF system, a user-friendly space mapping software engine, allowing automated SM optimization of microwave devices and circuits [10], [11].

Distributed evaluation of the fine model is realized through independent processing of the fine model responses corresponding to consecutive frequency samples using a number of machines. Thus, it can be applied for models using frequency-domain simulators. Because parallelization is implemented internally in the SMF system, it works regardless of whether the fine model simulator has a multi-processor analysis capability or not.

Fig. 1 shows the flowchart of the distributed fine model evaluation. Evaluation is performed by the main SMF copy and by $n$ distributed evaluation clients (SMFDs) running on separate processors. Suppose that the fine model is evaluated at $m$ frequency points, $f_{1}, f_{2}, \ldots, f_{m}$. This frequency sweep is divided into $K$ sub-bands, $B_{1}$ to $B_{K}$. In particular, the subbands may consist of single frequency samples. The information about the design variable vector $\boldsymbol{x}$ and frequency sub-bands is put into a so-called order set. Orders are picked up and processed by both the main SMF copy and by the SMFD clients and the results are exported into the results set, which is checked by the main SMF program. Once all orders are processed and corresponding responses are in the response set, the complete fine model response is returned.

Fig. 2 shows the architecture of the distributed model evaluation. All the information about the model, including the data allowing SMF and the SMFDs to prepare simulator input files, call the simulator and format the output data as well as the evaluation vector $\boldsymbol{x}$ and frequency sub-band, is gathered in the so-called order files. If SMF requests model evaluation, a number of order files corresponding to the number of frequency sub-bands as described before are generated and copied to a separate folder accessible by all SMFD clients. SMF and the SMFD clients pick up available order files and, after processing them, return the results to a result folder. Each SMFD client uses a separate working folder for temporary files. All the folders may reside in a designated directory on a local network drive or in a file system of a computational cluster. Communication between SMF, the SMFDs and the folders is realized through the SSH protocol.
In the ideal case, assuming that the main SMF program and $n$ SMFD clients are used in the distributed model evaluation process, the computation time should be $n+1$ times smaller than the evaluation time on a single processor. In practice this is never the case because of the following factors:

(i) In order to obtain maximum possible efficiency the number $K$ of frequency sub-bands should an integer multiplier of the number of processors $n+1$, which may not be the case;

(ii) The CPU type and speed, and, consequently, evaluation time of order files, may be different for different processors;

(iii) There is some overhead related to communication between SMF and the SMFDs and the designated folders;

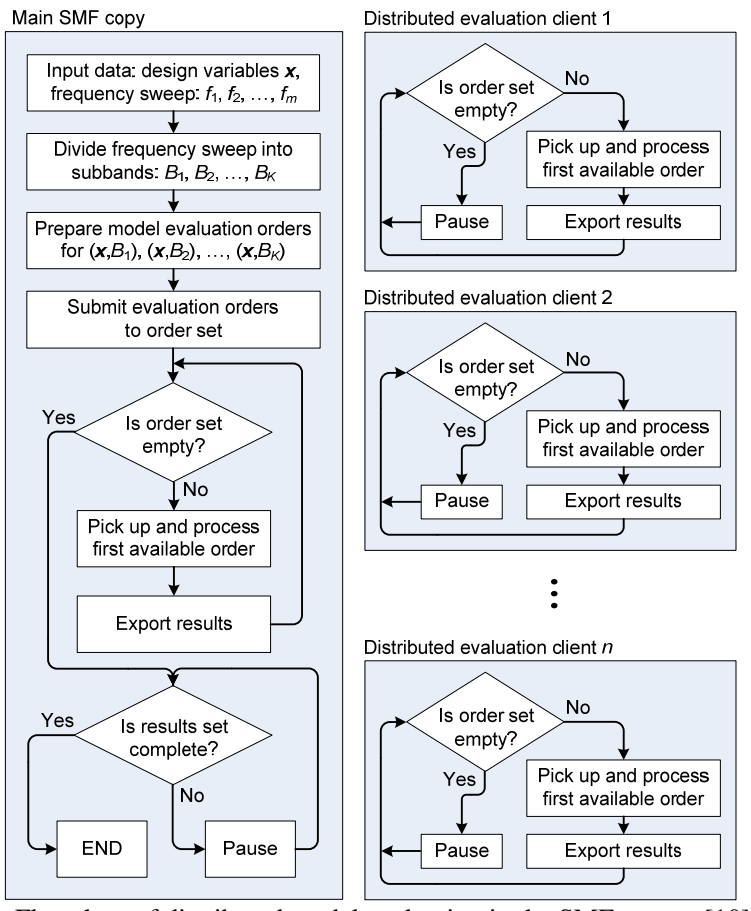

Fig. 1. Flowchart of distributed model evaluation in the SMF system [10].

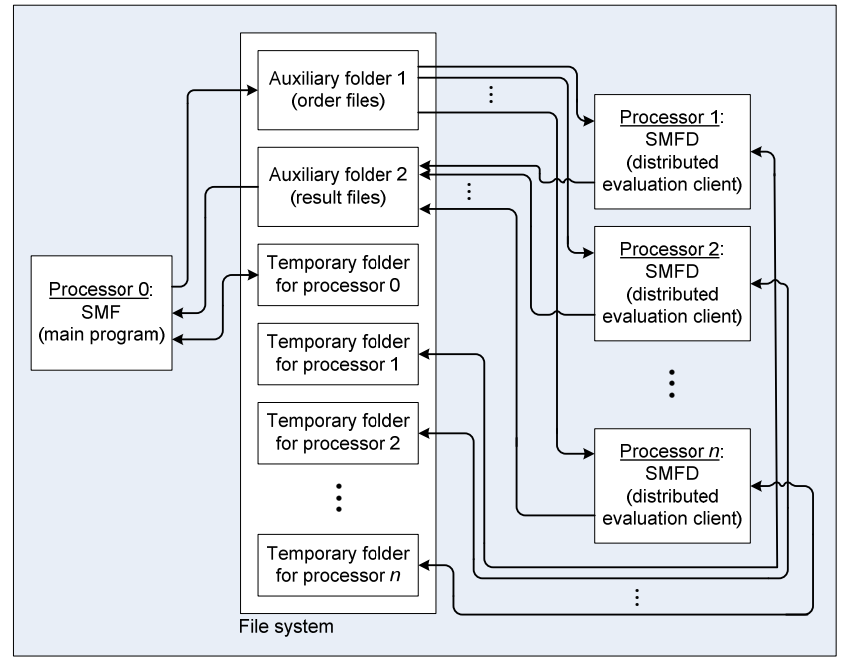

Fig. 2. Architecture of distributed model evaluation in the SMF system [10]. 
(iv) There may be additional overhead related to the fact that some actions which would normally be done once, e.g., meshing of the structure, might be performed for each frequency sub-band separately by each SMFD client;

The first factor plays the crucial role and the speed-up $s$ that can be obtained with our method, neglecting factors (ii), (iii) and (iv), is given by

$$
s=K /\lceil K /(n+1)\rceil
$$

where $\lceil$.$\rceil denotes a ceiling function.$

The parallel efficiency $\varepsilon$ is defined as the speed-up divided by the number of processors [12], i.e.,

$$
\varepsilon=s /(n+1)
$$

For example, if we have 30 sub-bands and 8 processors, the speed-up is 7.5 and the parallel efficiency is about $94 \%$.

In practice, because of factors (ii), (iii) and (iv), the actual parallel efficiency is smaller and typically it is between 60 and 90 percent, assuming that the number of processors is properly related to the number of frequency samples, i.e., the speed-up s (1) is sufficiently high, e.g., 90\% and more.

\section{EXAMPLES}

In this section we consider two examples of microwave design problems. To solve each problem we use a standard implementation of an SM algorithm, our SM algorithm with distributed fine model evaluation, as well as our SM algorithm with distributed fine model evaluation and inside-ADS parameter extraction and surrogate model optimization [9].

As the first example, consider the microstrip band-pass filter [13] shown in Fig. 3. The design parameters are $\boldsymbol{x}=\left[\begin{array}{lllll}L_{1} & L_{2} & L_{3} & L_{4} & g\end{array}\right]^{T}$. The fine model is simulated in FEKO [14] with a dense mesh (number of meshes about 700), the coarse model is the circuit model implemented in Agilent ADS [15] (Fig. 4). The design specifications are $\left|S_{21}\right| \leq-20 \mathrm{~dB}$ for $4.5 \mathrm{GHz} \leq \omega \leq 4.7 \mathrm{GHz}$ and $5.3 \mathrm{GHz} \leq \omega \leq 5.5 \mathrm{GHz}$, and $\left|S_{21}\right| \geq$ $-3 \mathrm{~dB}$ for $4.9 \mathrm{GHz} \leq \omega \leq 5.1 \mathrm{GHz}$. The number of frequency samples is $K=41$. The initial design is the coarse model optimal solution $\quad \boldsymbol{x}^{(0)}=\left[\begin{array}{lllll}6.784 & 4.890 & 6.256 & 5.28 & 0.0956\end{array}\right]^{T} \mathrm{~mm}$ (specification error $+24 \mathrm{~dB}$ ). We use the SM surrogate model with input and output SM of the form $\overline{\boldsymbol{R}}_{s}(\boldsymbol{x}, \boldsymbol{p})=$ $\overline{\boldsymbol{R}}_{s}(\boldsymbol{x}, \boldsymbol{c}, \boldsymbol{d})=\boldsymbol{R}_{c}(\boldsymbol{x}+\boldsymbol{c})+\boldsymbol{d}$. Fig. 5 shows the fine model initial and optimized responses after 4 SM iterations $\left(x^{(4)}=[6.4334 .743\right.$ $6.1724 .9110 .0787]^{T} \mathrm{~mm}$; the specification error is $-1.4 \mathrm{~dB}$ ).

Table I shows a comparison of the optimization time for the three implementations of the SM algorithm. For the standard implementation, most of the computational cost comes from the fine model evaluation (about 30 min per evaluation on a Pentium D $3.4 \mathrm{GHz}$ processor). Our SM algorithm with distributed fine model evaluation uses 14 processors (1 Pentium D $3.4 \mathrm{GHz}$ for SMF, and 13 nodes of the computational cluster containing Dual Core AMD $2 \mathrm{GHz}$ processors and Intel Xeon $3.06 \mathrm{GHz}$ processors for the SMFDs), which gives a very good speed-up (5) of 13.7 and a parallel efficiency of more than $97 \%$. The actual distributed evaluation time is about $2 \mathrm{~min} 30 \mathrm{~s}$, which gives a parallel efficiency of about $86 \%$. In this case the computational cost of solving the parameter extraction and surrogate optimization sub-problems is more than half of the total optimization cost. The application of inside-ADS parameter extraction and surrogate optimization [9] allows further reduction of the SM optimization cost to only 15 minutes, which is half the time necessary to evaluate the fine model on a single processor.

The second example is the band-stop microstrip filter with open stubs [16] shown in Fig. 6. The fine model is simulated with Sonnet's em [17] using a high-resolution grid with a $0.2 \mathrm{mil} \times 1 \mathrm{mil}$ cell size. The coarse model, Fig. 7 , is the equivalent circuit model implemented in Agilent ADS [15].

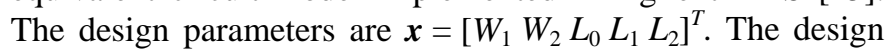
specifications are $\left|S_{21}\right| \leq 0.05$ for $9.4 \mathrm{GHz} \leq \omega \leq 10.6 \mathrm{GHz}$, and $\left|S_{21}\right| \geq 0.9$ for $5 \mathrm{GHz} \leq \omega \leq 8 \mathrm{GHz}$ and for $12 \mathrm{GHz} \leq \omega \leq$ $15 \mathrm{GHz}$. The number of frequency samples is $K=51$.

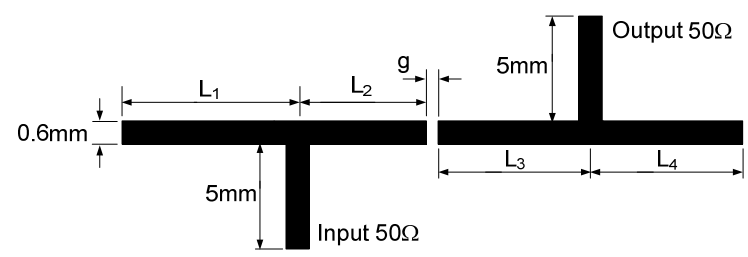

Fig. 3. Geometry of the microstrip band-pass filter [13].

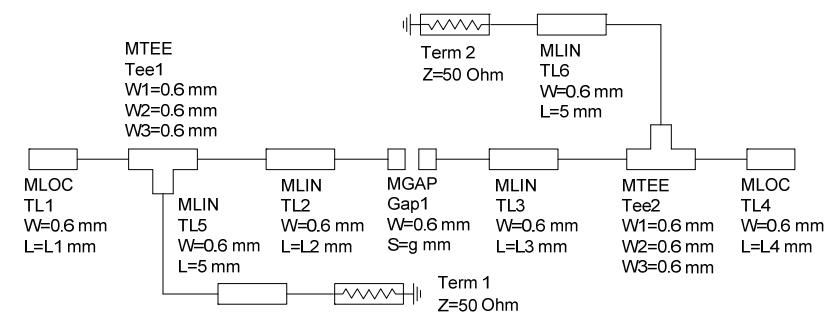

Fig. 4. Coarse model of the microstrip band-pass filter (Agilent ADS).

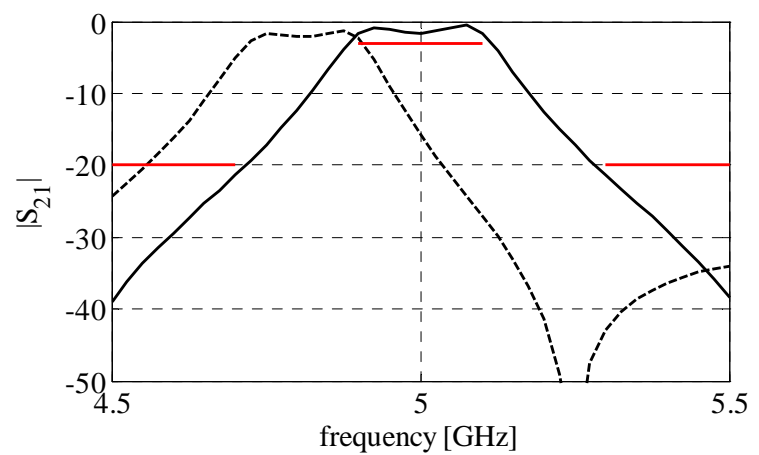

Fig. 5. Initial (dashed line) and optimized (solid line) $\left|S_{21}\right|$ versus frequency for the microstrip band-pass filter.

TABLE I

MicROSTRIP BANDPASS FILTER: OPTIMIZATION TIME FOR THE THREE IMPLEMENTATIONS OF SPACE MAPPING

\begin{tabular}{ccc}
\hline \hline SM Algorithm & $\begin{array}{c}\text { Optimization } \\
\text { Time }\end{array}$ & $\begin{array}{c}\text { Time } \\
\text { Savings }\end{array}$ \\
\hline Standard implementation & $169 \mathrm{~min}$ & - \\
Distributed fine model evaluation & $32 \mathrm{~min}$ & $81 \%$ \\
Distributed fine model evaluation and inside-ADS & $15 \mathrm{~min}$ & $91 \%$ \\
parameter extraction and surrogate optimization [9] & & \\
\hline \hline
\end{tabular}


The initial design is the coarse model optimal solution $\boldsymbol{x}^{(0)}=\left[\begin{array}{lllll}4.2 & 9.2 & 114.6 & 116 & 113\end{array}\right]^{T} \mathrm{~mm}$ (specification error $+0.024)$. We use the SM surrogate model with input and output space mapping of the form $\overline{\boldsymbol{R}}_{s}(\boldsymbol{x}, \boldsymbol{p})=$ $\overline{\boldsymbol{R}}_{\mathrm{s}}(\boldsymbol{x}, \boldsymbol{c}, \boldsymbol{d})=\boldsymbol{R}_{\mathrm{c}}(\boldsymbol{x}+\boldsymbol{c})+\boldsymbol{d}$. The fine model solution after $5 \mathrm{SM}$ iterations is $\boldsymbol{x}^{(5)}=\left[\begin{array}{lllll}3.6 & 11.6 & 116.2 & 122 & 107\end{array}\right]^{T} \mathrm{~mm}$ with a specification error of -0.02 .

Table II shows a comparison of the optimization time for the three implementations of the SM algorithm. For the standard implementation, most of the computational cost comes from the fine model evaluation (about 31 min per evaluation). The SM algorithm with distributed fine model evaluation uses 13 processors (1 Pentium D $3.4 \mathrm{GHz}$, and 12 nodes of the computational cluster). The distributed evaluation time is about $3 \mathrm{~min} 40 \mathrm{~s}$, which gives a parallel efficiency of about $65 \%$. In this case the computational cost of solving the parameter extraction and surrogate optimization sub-problems is over $60 \%$ of the total optimization cost. As in the previous example, the application of inside-ADS parameter extraction and surrogate optimization [9] allows further reduction of the SM optimization cost to only 25 minutes, which is less than necessary to evaluate the fine model on a single processor.

\section{CONCLUSION}

A new implementation of our SM algorithm with distributed evaluation of the fine model is presented. The new algorithm allows substantial reduction of the SM optimization time in comparison with the standard implementation. When combined with previously published techniques for the reduction of the parameter extraction and surrogate optimization cost, it permits us to complete SM optimization in less time than necessary to evaluate the fine model on a single processor.

\section{ACKNOWLEDGEMENT}

The authors thank Dr. J.C. Rautio of Sonnet Software, Inc., for providing the multiple licenses needed to carry out this work.

\section{REFERENCES}

[1] J.W. Bandler, Q.S. Cheng, S.A. Dakroury, A.S. Mohamed, M.H. Bakr, K. Madsen, and J. Søndergaard, "Space mapping: the state of the art," IEEE Trans. Microwave Theory Tech., vol. 52, no. 1, pp. 337-361, Jan. 2004.

[2] J.W. Bandler, Q.S. Cheng, N.K. Nikolova, and M.A. Ismail, "Implicit space mapping optimization exploiting preassigned parameters," IEEE Trans. Microwave Theory Tech., vol. 52, no. 1, pp. 378-385, Jan. 2004.

[3] S. Koziel, J.W. Bandler, and K. Madsen, "A space mapping framework for engineering optimization: theory and implementation," IEEE Trans. Microwave Theory Tech., vol. 54, no. 10, pp. 3721-3730, Oct. 2006.

[4] M. Redhe and L. Nilsson, "Optimization of the new Saab 9-3 exposed to impact load using a space mapping technique," Structural and Multidisciplinary Optimization, vol. 27, pp. 411-420, 2004.

[5] S. Amari, C. LeDrew, and W. Menzel, "Space-mapping optimization of planar coupled-resonator microwave filters," IEEE Trans. Microwave Theory Tech., vol. 54, no. 5, pp. 2153-2159, May 2006.

[6] H.-S. Choi, D. H. Kim, I. H. Park, and S. Y. Hahn, "A new design technique of magnetic systems using space mapping algorithm," IEEE Trans. Magn., vol. 37, no. 5, pp. 3627-3630, Sept. 2001.

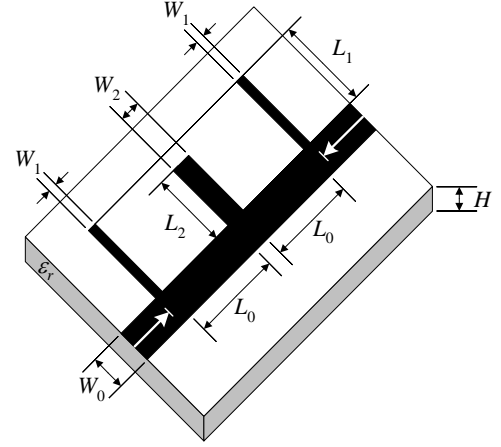

Fig. 6. Geometry of the band-stop microstrip filter with open stubs [16].

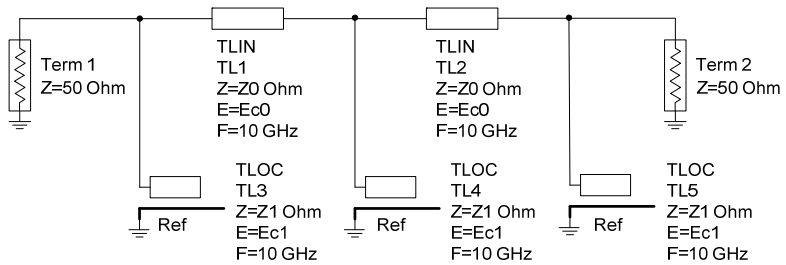

Fig. 7. Coarse model of the band-stop microstrip filter (Agilent ADS).

TABLE I

MicROSTRIP BANDPASS FILTER: OPTIMIZATION TIME FOR THE THREE IMPLEMENTATIONS OF SPACE MAPPING

\begin{tabular}{ccc}
\hline \hline SM Algorithm & Optimization & Time \\
& Time & Savings \\
\hline Standard implementation & $220 \mathrm{~min}$ & - \\
Distributed fine model evaluation & $56 \mathrm{~min}$ & $75 \%$ \\
Distributed fine model evaluation and inside-ADS & $25 \mathrm{~min}$ & $89 \%$ \\
parameter extraction and surrogate optimization [9] & & \\
\hline \hline
\end{tabular}

[7] S. Koziel and J.W. Bandler, "Controlling convergence of space-mapping algorithms for engineering optimization," Int. Symp. Signals, Systems and Electronics, URSI ISSSE 2007, Montreal, Canada, 2007, pp. 21-23.

[8] S. Koziel and J.W. Bandler, "Interpolated coarse models for microwave design optimization with space mapping," IEEE Trans. Microwave Theory Tech., vol. 55, no. 8, pp. 1739-1746, Aug. 2007.

[9] S. Koziel, Q.S. Cheng, and J.W. Bandler, "Improving efficiency of space mapping optimization of microwave structures and devices,” IEEE MTT-S Int. Microwave Symp. Dig, Honolulu, HI, 2007, pp. 1995-1998.

[10] S. Koziel and J.W. Bandler, "SMF: a user-friendly software engine for space-mapping-based engineering design optimization," Int. Symp. Signals, Systems and Electronics, URSI ISSSE 2007, Montreal, Canada, 2007, pp. 157-160.

[11] SMF, Bandler Corporation, P.O. Box 8083, Dundas, ON, Canada L9H 5E7, 2006.

[12] L. Zhang, Y. Cao, S. Wan, H. Kabir, and Q.J. Zhang, "Parallel automatic model generation technique for microwave modeling," IEEE MTT-S Int. Microwave Symp. Dig, Honolulu, HI, 2007, pp. 103-106.

[13] A. Hennings, E. Semouchkina, A. Baker, and G. Semouchkin, "Design optimization and implementation of bandpass filters with normally fed microstrip resonators loaded by high-permittivity dielectric," IEEE Trans. Microwave Theory Tech., vol. 54, no. 3, pp. 1253-1261, March 2006.

[14] FEKO ${ }^{\circledR}$ User's Manual, Suite 4.2, June 2004, EM Software \& Systems-S.A. (Pty) Ltd, 32 Techno Lane, Technopark, Stellenbosch, 7600, South Africa.

[15] Agilent ADS, Version 2003C, Agilent Technologies, 1400 Fountaingrove Parkway, Santa Rosa, CA 95403-1799, 2003.

[16] M.H. Bakr, J.W. Bandler, M.A. Ismail, J.E. Rayas-Sánchez, and Q.J. Zhang, "Neural space-mapping optimization for EM-based design," IEEE Trans. Microwave Theory Tech., vol. 48, no. 12, pp. 2307-2315, Dec. 2000.

[17] $\boldsymbol{e m}^{\mathrm{TM}}$ Version 10.52, Sonnet Software, Inc., 100 Elwood Davis Road, North Syracuse, NY 13212, USA. 